\title{
The Vacuum Phototriodes for the CMS Electromagnetic Calorimeter
}

\section{R M Brown*†, K W Bell, D J A Cockerill, P S Flower, B W Kennedy, A L Lintern, $\mathrm{M}$ Sproston and $\mathbf{J} \mathbf{H}$ Williams}

CLRC Rutherford Appleton Laboratory, Didcot, Oxfordshire, OX11 OQX, UK

E-mail: :

\section{P R Hobson, D C Imrie and O Sharif}

Department of Electronic and Computer Engineering, Brunel University, Uxbridge, Middlesex, UB8 3PH, UK

Abstract: The measurement of scintillation light from the lead tungstate crystals of the CMS ECAL poses a substantial technical challenge, particularly in the Endcap regions, where the radiation levels are highest. The photodetectors must be fast, sensitive, radiation-hard, and operate with significant internal gain in a magnetic field of 4 Tesla. The measured performance characteristics of 500 pre-production VPTs, developed to satisfy the needs of CMS, are presented.

\section{Introduction}

The electromagnetic calorimeter (ECAL) [1] [1] scintillating crystal as the active medium. This is the first application of this material in particle physics. In many respects, lead tungstate is an ideal crystal for use at the LHC. It has:

- a very short radiation length $(8.9 \mathrm{~mm})$ and Molière radius $(21.9 \mathrm{~mm})$, allowing a compact design

- fast scintillation emission ( $>90 \%$ of light emitted within $100 \mathrm{~ns}$ ) in the visible region $(\sim 420 \mathrm{~nm})$

- strong resistance to radiation induced darkening through the formation of stable colour centres.

\footnotetext{
${ }^{*}$ Speaker.

${ }^{\dagger}$ We wish to thank H F Heath and colleagues at Bristol University, UK, and D Seliverstov and colleagues at PNPI, Russia.
} 


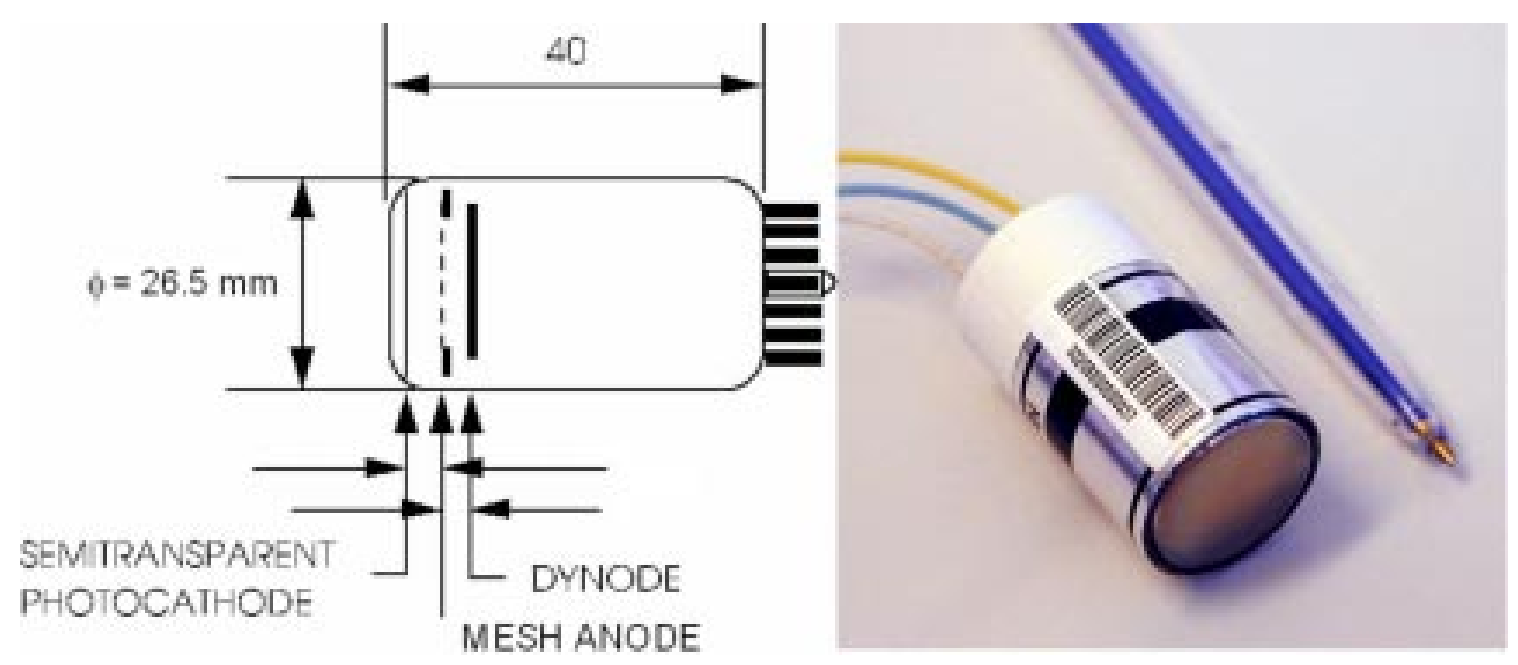

Figure 1: A vacuum phototriode with a nominal external diameter of 1 inch, supplied by Research Institute Electron for the CMS Electromagnetic Calorimeter Endcaps.

However, the light yield is rather low $(\sim 50$ photons $/ \mathrm{MeV})$, necessitating the use of photodetectors with internal gain. Furthermore, in CMS, the ECAL is located within the magnetic field volume of the superconducting solenoid. Thus the photodetectors are required to operate in a $4 \mathrm{~T}$ magnetic field.

The ECAL comprises two subsystems: the 'Barrel' ( $\sim 61000$ crystals $)$ covering the pseudorapidity range $|\eta|<1.48$, and a pair of 'Endcaps' ( $\sim 15000$ crystals) covering the range $1.48<|\eta|<3.0$. The photodetectors chosen for the Barrel are avalanche photodiodes

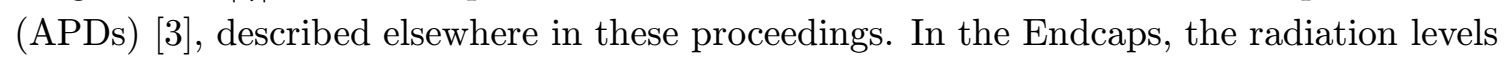
are much higher than in the Barrel, precluding the use of silicon-based photodetectors. The solution chosen in this region is therefore to use vacuum phototriodes (VPTs). The magnetic field orientation is favourable for the use of such detectors in the Endcaps, but not in the Barrel.

\section{Application of VPTs in CMS}

A vacuum phototriode is essentially a photomultiplier tube with a single stage of gain (figure 1). Optical photons strike a semi-transparent photocathode deposited on the inner surface of the front window. The emitted photoelectrons are accelerated towards the anode, which consists of a very fine metal mesh. A fraction ( $50 \%)$ of the electrons pass through the anode and strike the solid metal dynode, located just behind the anode. Secondary electrons ejected from the dynode are accelerated back towards the anode, where most are collected. The dynode surface is coated with the same bialkali material as that forming the photocathode and under operating conditions $\left(V_{A}=1000 \mathrm{~V}, V_{D}=800 \mathrm{~V}\right)$ has a secondary emission coefficient of approximately 20. Thus, taking into account the transparency of the anode mesh, the effective gain of the VPT is of order 10. A more complete discussion of the operation is given elsewhere [in]. 

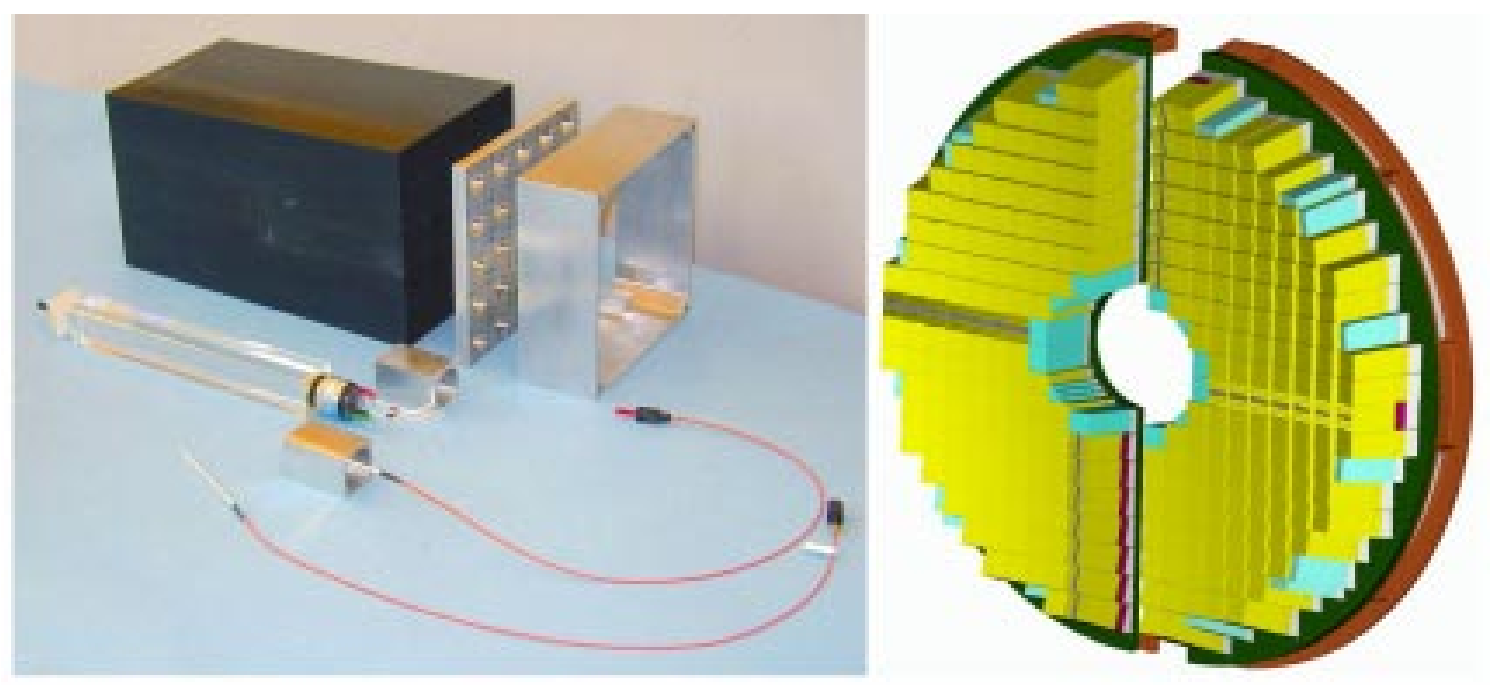

Figure 2: Details of the construction of the ECAL Endcaps. On the left, elements of a 'supercrystal' (a module of 25 crystals): mechanical components, a crystal/VPT assembly, monitoring system fibres. On the right, the arrangement of supercrystals on a pair of 'Dees'.

The Endcap crystals have a square back face with dimensions $30 x 30 \mathrm{~mm}^{2}$. They are grouped in 'supercrystals' consisting of $5 \times 5$ crystals housed in a thin walled 'alveola' structure made from carbon fibre composite material. An Endcap consists of two 'Dees', each of which contains 150 supercrystals. Figure 2 shows the components of a supercrystal, and the arrangement of supercrystals on an Endcap. The VPTs developed for CMS have an outer diameter of $26.5 \mathrm{~mm}$ - the largest dimension that could be accommodated within the mechanical constraints of the supercrystal. The effective photocathode diameter is $19 \mathrm{~mm}$, providing adequate coverage of the crystal face. The crystals are slightly tapered along their length, to give a projective geometry. The angle of the crystal axis (and hence the VPT axis) with respect to the direction of the magnetic field varies from $5.7^{\circ}$ at the inner edge of the Endcap to $25.6^{\circ}$ at the outer circumference.

Following several years of intensive R\&D [i $[$ i ], a tender exercise was conducted with potential producers, resulting in the placement of an order for 500 'pre-production' devices with Research Institute Electron (RIE) in St. Petersburg. Delivery was completed at the end of 2000 .

\section{Performance Characteristics}

Samples of the pre-production tubes have been evaluated at Brunel University, both at zero magnetic field, and at $4 \mathrm{~T}$ field with a fixed tube orientation of $15^{\circ}$ with respect to the field. All the devices have been measured at Rutherford Appleton Laboratory at several magnetic field values up to $1.8 \mathrm{~T}$ and over a range of angles from $-30^{\circ}$ to $+30^{\circ}$. Figure 3 shows how the anode response changes as a function of the angular orientation in a magnetic field. The oscillatory structure at small angles is associated with the periodic pattern of 

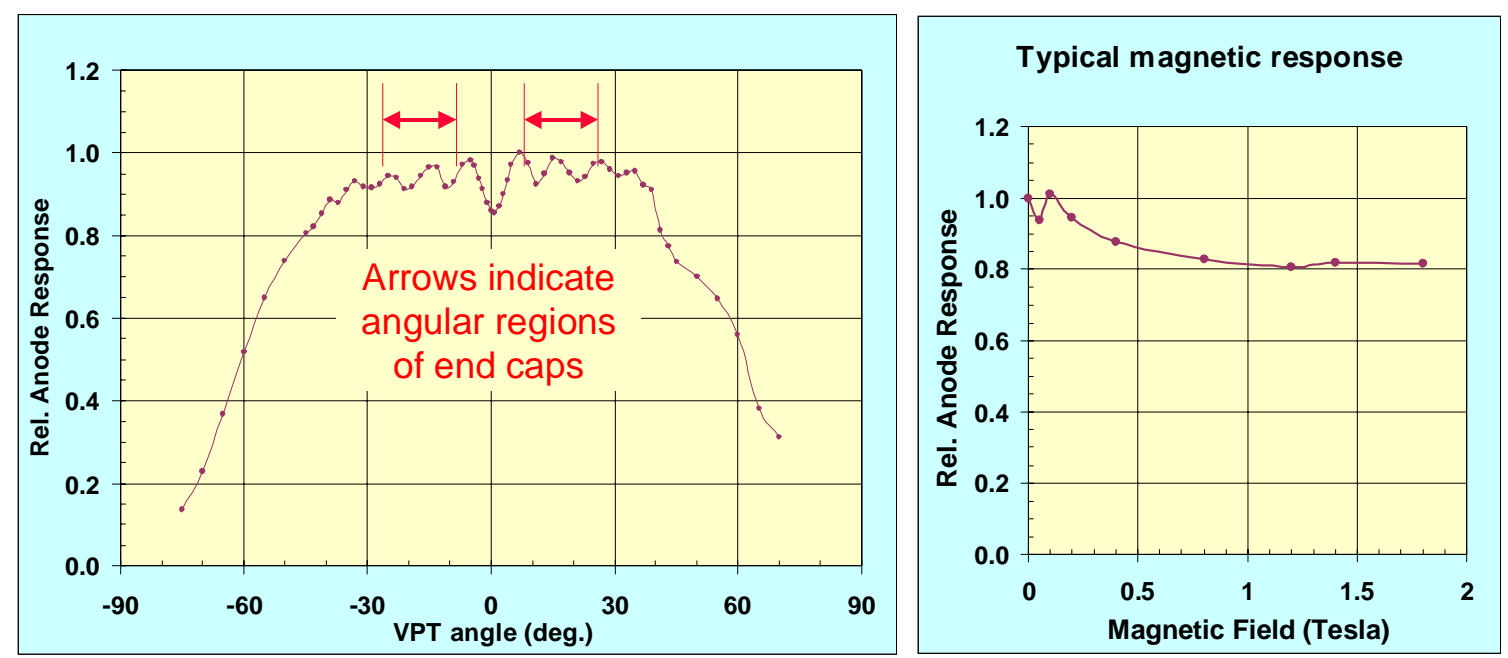

Figure 3: The behaviour of a typical VPT in a magnetic field. On the left, the anode response as a function of the angle between the VPT axis and field direction for a field of $1.8 \mathrm{~T}$. On the right, the anode response as a function of field strength for a VPT/field-angle of $15^{\circ}$.

the anode mesh. At angles beyond $\pm 45^{\circ}$, the response falls rapidly as photoelectrons strike the walls of the glass envelope before reaching the anode and dynode. The variation in anode response as a function of magnetic field strength is also shown in figure 3 . The output displays significant field dependence below $1 \mathrm{~T}$, but at higher fields the response changes very slowly. The fixed field measurements at Brunel confirm that the performance is maintained up to $4 \mathrm{~T}$.

The measured distribution of anode response (at $1.8 \mathrm{~T}$ and $15^{\circ}$ ) is shown in figure 4 (left). The entries have been separated into three classes corresponding to the start, middle and end of the production run. There is a clear indication that the average response tended to improve through the course of the manufacturing run. The proof-of-principle that the $\mathrm{PbWO}_{4}$ - VPT combination could meet the performance requirements of the CMS ECAL was demonstrated using a 5x5 test array of crystals in a high energy electron beam at

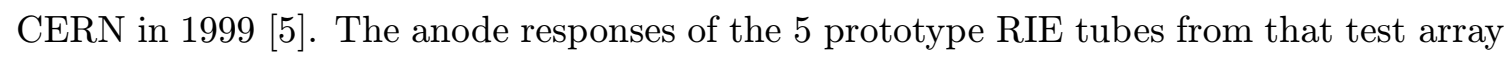
have also been measured on the test set-up at RAL. They appear as the open entries in figure 4. It is encouraging to note that the average performance of the pre-production tubes is substantially better than that of the prototype devices.

\section{Radiation hardness}

The main susceptibility of vacuum photodetectors to damage by radiation arises from darkening of the window caused by the formation of colour centres. Tubes with a standard borosilicate window are quite readily degraded in this way. Radiation hard tubes can be realised by incorporating a fused silica window, but these would be prohibitively expensive for application in CMS. A good compromise is to make the window from ultraviolet transmitting glass, which is much more radiation resistant than borosilicate glass, yet adds little 

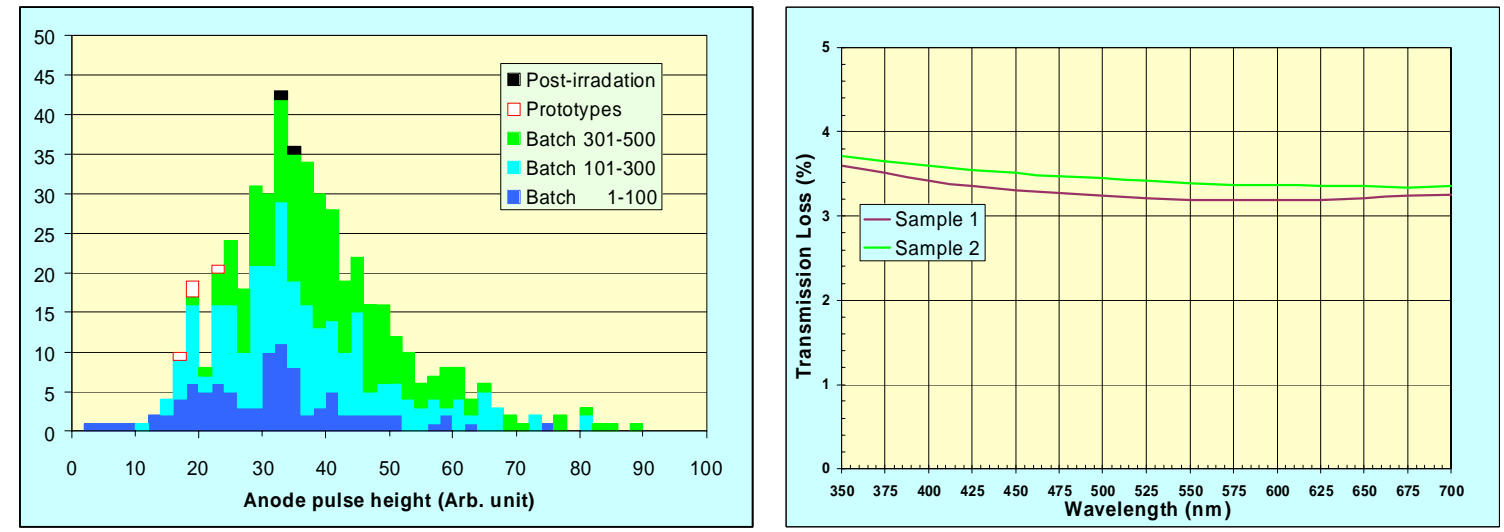

Figure 4: The histogram on the left shows the distribution of anode responses for 500 preproduction VPTs (shaded) and 5 earlier prototype devices (unshaded). The anode responses of two pre-production tubes, after ${ }^{60} \mathrm{Co}$ irradiation to $25 \mathrm{kGy}$, are also shown (black). The curves on the right show the internal optical transmission as a function of wavelength for two faceplate samples, after ${ }^{60} \mathrm{Co}$ irradiation to $25 \mathrm{kGy}$.

to the cost of the tube. Measurements on face plate samples using the ${ }^{60} \mathrm{Co}$ irradiation facility at Brunel show that at doses up to $25 \mathrm{kGy}$ (the expected dose at $|\eta|=2.6$, after 10 years of LHC operation) the loss in transmission in the region of $\mathrm{PbWO}_{4}$ emission is less than $4 \%$ (figure 4 (right)). Complete tubes have also been irradiated while under bias. The anode responses of two irradiated pre-production tubes are indicated by the black entries in figure 4 (left). The post-irradiation performance is typical of the unirradiated devices.

\section{Summary}

High-field, radiation-resistant VPTs have been developed to fulfil the requirements of the CMS ECAL Endcaps. Test measurements on 500 pre-production devices show that the design specifications can be met.

\section{References}

[1] The CMS Electromagnetic Calorimeter Project Technical Design Report, CERN/LHCC 97-33 (1997).

[2] The Compact Muon Solenoid Technical Proposal, CERN/LHCC 94-38 (1994).

[3] K. Deiters et al., Properties of the avalanche photodiodes for the CMS electromagnetic

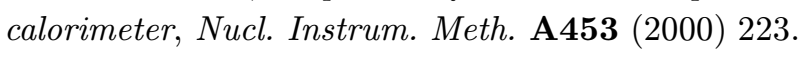

[4] K.W. Bell et al., The Development of Vacuum Phototriodes for the CMS Electromagnetic Calorimeter, 'Nucl. Instrum. Meth. A469 $(200 \overline{1}) 2 \overline{9}_{\mathrm{r}}^{\prime}$

[5] M. Appollonio et al., Test Results from a Prototype Lead Tungstate Crystal Calorimeter with Vacuum Phototriode Readout for the Compact Muon Solenoid Experiment,

Nucl. Instrum. Meth., to be published. 